\title{
The Effect of Platelet-Rich Plasma on the Bone Healing around Calcium Phosphate-Coated and Non-coated Oral Implants in Trabecular Bone
}

\author{
DIMITRIS NIKOLIDAKIS, D.D.S., ${ }^{1}$ JULIETTE VAN DEN DOLDER, Ph.D., ${ }^{1}$ JOOP G.C. \\ WOLKE, Ph.D., ${ }^{1}$ PAUL J.W. STOELINGA, D.D.S., M.D., Ph.D., ${ }^{2}$ \\ and JOHN A. JANSEN, D.D.S., Ph.D. ${ }^{1}$
}

\begin{abstract}
The effect of local application of autologous platelet-rich plasma (PRP) on bone healing in combination with the use of titanium implants with 2 different surface configurations was investigated. PRP fractions were obtained from venous blood sample of 6 goats and applied via gel preparation and subsequent installation in the implant site or via dipping of the implant in PRP liquid before insertion. Thirty-six implants (18 non-coated and 18 calcium phosphate $(\mathrm{CaP})$ coated) were placed into the goat femoral condyles (trabecular bone). The animals were sacrificed at 6 weeks after implantation, and implants with surrounding tissue were processed for light microscopical evaluation. In addition to subjective description of the histological findings, histomorphometrical variables were also evaluated (the boneimplant contact and the bone mass adjacent to the implant). Significantly more interfacial bone-to-implant contact was observed for all 3 groups of CaP-coated implants and the titanium/liquid group (non-coated implant with PRP liquid) than for the other 2 non-coated titanium groups (with PRP gel or without PRP). The evaluation of the bone mass close to implant surface indicated that all the groups induced a significant increase of the bone mass except the PRP gel groups. On the basis of the observations, it was concluded that magnetron-sputtered CaP coatings can improve the integration of oral implants in trabecular bone. The additional use of PRP did not offer any significant effect on the bone response to the CaP-coated implants, whereas PRP in a liquid form showed a significant effect on bone apposition to roughened titanium implants during the early post-implantation healing phase.
\end{abstract}

\section{INTRODUCTION}

A MAJOR ADVANCE IN DENTISTRY has been the replacement of lost natural teeth using oral implants. The predictability and long-term success of oral implants are well documented. ${ }^{1,2}$ On the other hand, the success rates have been reported to vary between patients, and several factors, such as implant design, implant surface, surgical technique, bone type, and loading conditions, have been shown to influence implant osseointegration. ${ }^{3}$ Numerous studies have shown that the success rate of implants in local host bone with poor osteo-regenerative potential is low. ${ }^{4}$ In view of this, Lekholm and Zarb have described 4 types of bone density (I-IV). ${ }^{5}$ The survival rate of oral implants placed into type IV bone is dramatically lower than with the other types. ${ }^{6}$ Furthermore, clinical experience has shown that implant failure occurs at relatively early stages after surgery.

Considering the effect of implant surface characteristics on the bone response, ${ }^{7} 2$ different categories of qualities can be discerned: topography and physicochemistry. The topographic features of the implant surface are related to the degree of surface roughness. Moderate values of surface

\footnotetext{
${ }^{1}$ Department of Periodontology and Biomaterials, Radboud University Nijmegen Medical Center, Nijmegen, The Netherlands.

${ }^{2}$ Department of Oral Maxillofacial Surgery, Radboud University Nijmegen Medical Center, Nijmegen, The Netherlands.
} 
roughness (Sa: $1-2 \mu \mathrm{m})$ evoke a greater bone response than lower or higher values. ${ }^{7}$ The use of calcium phosphate $(\mathrm{CaP})$ coatings has also been shown to benefit the bone response during the initial healing period after implant insertion. ${ }^{8}$ Various animal studies have proved that CaP-coated implants show greater bone contact than non-coated implants. ${ }^{9,10}$

In addition to surface quality, growth factors can also stimulate bone healing around implants. The effect of osteoinductive factors like bone morphogenetic proteins or transforming growth factor (TGF)- $\beta$ has been a topic of various experiments. ${ }^{11,12}$ The disadvantage of these growth factors is that they are expensive and concerns exists about their safety in human administration. ${ }^{13}$ Alternatively, platelet-rich plasma (PRP), which is a volume of autogenous plasma that has a platelet concentration above baseline, is a proven source of growth factors. ${ }^{14}$ Growth factors released from platelets ${ }^{15}$ include platelet-derived growth factor (PDGF), TGF- $\beta$, platelet-derived epidermal growth factor (PDEGF), platelet-derived angiogenesis factor (PDAF), insulin-like growth factor I (IGF-I), and platelet factor 4 (PF-4). The positive effect of PRP is attributed to the angio-genetic, cell-proliferative, and differentiating influence of TGF- $\beta$ and PDGF, which are present in PRP in high concentrations. ${ }^{16}$ Because PRP is developed from autologous blood, it is inherently safe and free from transmissible diseases. This distinguishes it from recombinant growth factors that are not native. ${ }^{17}$ PRP has been mainly used as a bone graft-enhancement material, and thus there is limited evidence to support the use of PRP in combination with dental implant placement. ${ }^{18,19}$

Furthermore, PRP is usually applied as a gel by mixing it immediately before use with calcium chloride and bovine thrombin. However, the use of bovine thrombin has been reported to be associated with the development of antibodies to factors $\mathrm{V}$ and XI, which can result in the risk of lifethreatening coagulopathies. ${ }^{20}$ Therefore, Dugrillon et al. have proposed the formation of PRP gel by adding calcium without using thrombin as activator. ${ }^{21}$

Therefore, the purpose of the present study was to investigate the effect of local application of autologous PRP on the trabecular bone healing around oral implants with 2 different surface qualities: a CaP-coated surface and a noncoated titanium surface with similar roughness. In addition, PRP was applied in 2 ways: using a gel preparation by mixing the PRP fraction with calcium chloride solution and bovine thrombin followed by subsequent installation in the implant site and dipping the implant into the PRP fraction before its insertion without using any additional agent.

\section{MATERIALS AND METHODS}

\section{Implant preparation}

Thirty-six cylindrical, commercially pure titanium implants with a diameter of $3.4 \mathrm{~mm}$ and a length of $9 \mathrm{~mm}$ were used. They were grit-blasted with aluminum oxide particles, acid-etched with a mixture of hydrochloric acid and sulfuric acid for $1 \mathrm{~min}$, cleaned ultrasonically in isopropanol, and dried at $100^{\circ} \mathrm{C}$. Subsequently, half of the implants were provided with a thin $\mathrm{CaP}$ coating.

The $\mathrm{CaP}$ coatings were applied using a commercially available radio frequency magnetron sputter deposition system (Edwards ESM 100, Sussex, UK). The thickness of the $\mathrm{CaP}$ sputter coating was $1 \mu \mathrm{m}$. After deposition, the coated specimens were subjected to an additional heat treatment for $2 \mathrm{~h}$ at $550^{\circ} \mathrm{C}$.

The physicochemical analysis showed that the heattreated coatings had a crystalline apatite-like structure with a calcium:phosphate ratio of 1.8:2.0. Surface roughness measurements, using a universal surface tester (Innowep $\mathrm{GmbH}$, Würzburg, Germany), revealed that the roughness value for the non-coated implants was $2.1 \mu \mathrm{m}$, whereas the CaP-coated implants showed a roughness value of $2.3 \mu \mathrm{m}$. Scanning electron microscopy revealed that the non-coated titanium implants had a surface morphology with sharp peaks, whereas the surface of the CaP-coated implants had a smooth globular appearance (Fig. 1).

Finally, all implants were cleaned ultrasonically for 10 min to remove any loose particles and sterilized in an autoclave.

\section{Procedure of PRP preparation and application}

The PRP fraction was obtained from venous blood of goats 1 day before implant placement; $250 \mathrm{~cm}^{3}$ of blood was drawn and centrifuged at a transfusion laboratory (SanQuin, Nijmegen, The Netherlands) in various cycles. $^{22}$ Finally, $7.5 \mathrm{~mL}$ of PRP suspension was obtained (Table 1). Counting of the platelet concentration was also performed (platelet count exceeding $800 \times 10^{6} / \mathrm{mL}$ ).

The final application of the PRP fraction was performed using gel preparation and subsequent installation in the implant site or dipping of the implant in PRP liquid before insertion. The PRP gel was obtained at the time of surgery by mixing the PRP fraction with $10 \%$ calcium chloride solution and 300 IU of bovine thrombin (Fibriquick, Organon Teknika, Boxtel, The Netherlands). The PRP liquid was used alone, without addition of any agent.

\section{Animal model and implantation procedure}

Six healthy, mature female Saanen goats, weighing about $60 \mathrm{~kg}$, were used in this study. Before surgery, blood samples were taken from the goats to ensure that they were free of caprine arthritis encephalitis and caseous lymphadenitis. The goats were housed in a stable, and national guidelines for care and use of laboratory animals were observed. The surgical procedure was performed under general anesthesia, which was induced using an intravenous injection of pentobarbital and maintained using isoflurane $2 \%$ to $3 \%$ through a constant volume ventilator via an 

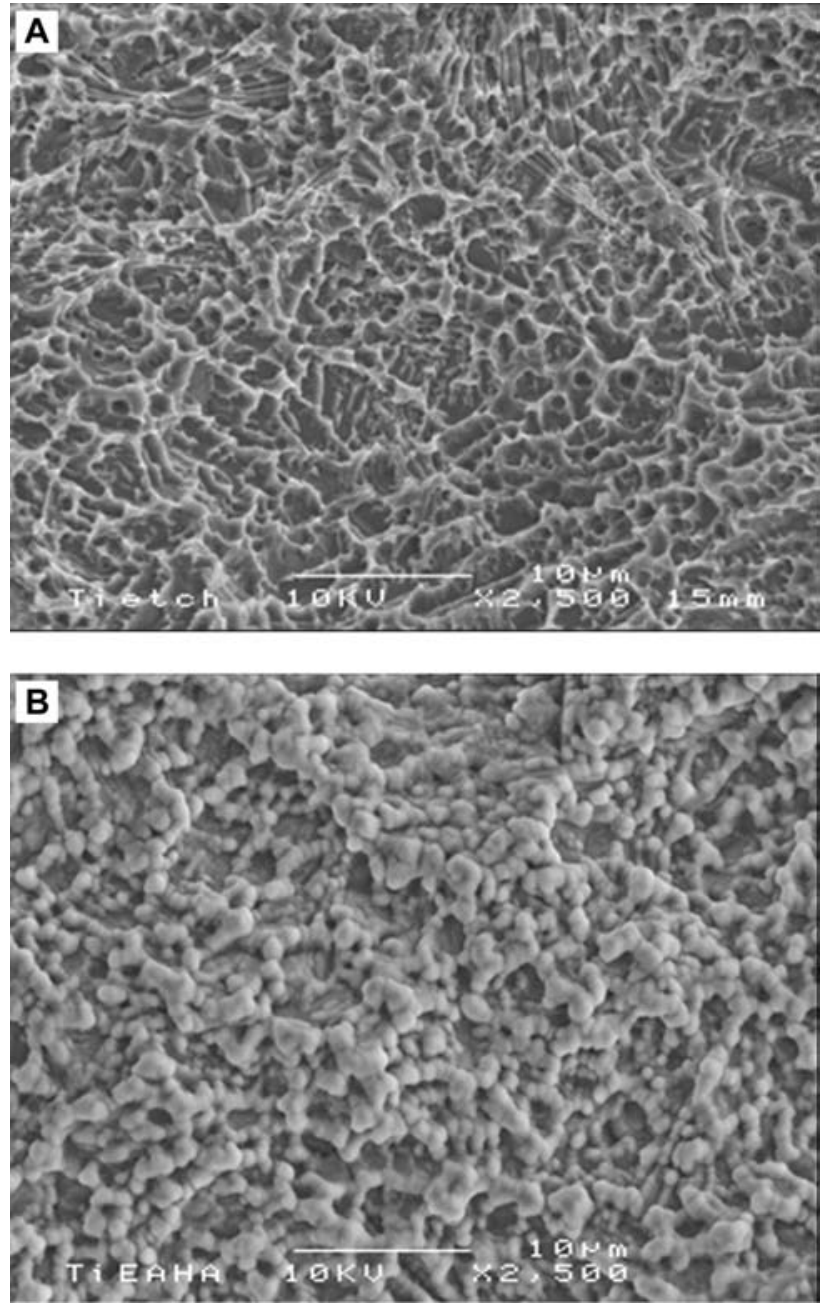

FIG. 1. The final roughness value for the non-coated implants was $2.1 \mu \mathrm{m}$ (A), whereas the final roughness value for the calcium phosphate-coated implants was $2.3 \mu \mathrm{m}(\mathbf{B})$.

endotracheal tube. During surgery, the goats were connected to a heart monitor.

They also received antibiotic prophylaxis to reduce perioperative infection risk, as follows:

- During surgery: Albipen 15\%, $3 \mathrm{~mL} / 50 \mathrm{~kg}$ subcutaneously (sc)

- 1 day after surgery: Albipen LA, $7.5 \mathrm{~mL} / 50 \mathrm{~kg} \mathrm{sc}$

- 3 days after surgery: Albipen LA, $7.5 \mathrm{~mL} / 50 \mathrm{~kg} \mathrm{sc}$

The implants were inserted into the left and right distal femoral condyles of the goat. For the insertion of the implants, each animal was immobilized on its back, and its hind legs were shaved, washed, and disinfected with povidoneiodine. A longitudinal incision was made on the medial surface of the left and right femur, and the medial condyle was exposed. After exposure of the femoral condyle, 3 pilot holes were drilled $(1.6 \mathrm{~mm})$, which were gradually widened with different drills to the final diameter of the implant $(3.4 \mathrm{~mm})$. The distance between the 3 drill holes was always
Table 1. Volume and Platelet Concentration of Platelet-Rich Plasma (PRP) Harvested FROM EACH GOAT

\begin{tabular}{lcc}
\hline Goat & PRP volume & Platelet count \\
\hline 1 & $7.5 \mathrm{~mL}$ & $1200 \times 10^{6} / \mathrm{mL}$ \\
2 & $7.5 \mathrm{~mL}$ & $800 \times 10^{6} / \mathrm{mL}$ \\
3 & $7.5 \mathrm{~mL}$ & $1000 \times 10^{6} / \mathrm{mL}$ \\
4 & $7.5 \mathrm{~mL}$ & $800 \times 10^{6} / \mathrm{mL}$ \\
5 & $7.5 \mathrm{~mL}$ & $1000 \times 10^{6} / \mathrm{mL}$ \\
6 & $7.5 \mathrm{~mL}$ & $1200 \times 10^{6} / \mathrm{mL}$ \\
\hline
\end{tabular}

at least $1 \mathrm{~cm}$. The bone preparation was performed with a gentle surgical technique using a low rotational drilling speed (maximum $450 \mathrm{rpm}$ ) and continuous internal cooling. After bone preparation, the holes were irrigated with sterile water. After press-fit insertion of the implants, the soft tissues were closed in separate layers using resorbable Vicryl 3-0 sutures. Thirty-six implants were placed: $18 \mathrm{CaP}$-coated and 18 non-coated implants. Each animal received 6 implants: 3 CaP-coated implants in 1 femoral condyle and 3 non-coated implants in the other. Regarding the use of PRP, PRP gel was applied in 2 goats, PRP liquid fraction via dipping of the implant was applied in 2 other goats, and the residual 2 goats did not receive any PRP application (control group). This resulted in 6 different treatment groups: (1) CaP-coated implants $(\mathrm{CaP}),(2) \mathrm{CaP}-$ coated implants + PRP liquid (CaP/liquid), (3) CaP-coated implants + PRP gel (CaP/gel), (4) non-coated implants (titanium), (5) noncoated implants + PRP liquid (titanium/ liquid), and (6) noncoated implants + PRP gel (titanium/gel).

Postoperatively, the animals were housed in a stable, where they could move freely. The animals were sacrificed at 6 weeks after implantation using an overdose of Nembutal, and the implants and surrounding tissue were retrieved for histological preparation.

\section{Histological preparation}

After sacrifice of the animals, the femoral distal condyles were excised, and excess tissue was removed. Then, using a diamond saw, the retrieved condyles were divided into smaller specimens suitable for histological processing. Each specimen included 1 implant with surrounding bone. The samples were fixed in $4 \%$ formaldehyde. Subsequently, they were dehydrated through a graded series of ethanol, embedded in methylmethacrylate, and polymerized. Longitudinal, mid-implant, thin non-decalcified sections $(10 \mu \mathrm{m})$ were made with a modified diamond blade-sawing microtome technique. The sections were stained with methylene blue and basic fuchsin and examined for histological and histomorphometrical evaluation.

\section{Histological and histomorphometrical evaluation}

The histological evaluation was performed using a light microscope (Leica Microsystems GmbH, Wetzlar, Germany) 
and included a description of the observed tissue response. In addition, microscopic images were projected with a magnification of $1.6 \times$ on a color monitor, and a digital image analysis software (Leica Qwin Pro-image analysis system, Wetzlar, Germany) was used for the histomorphometrical measurements.

The histomorphometrical analysis consisted of:

(a) Bone contact percentage: The amount of bone contact was defined as the percentage of implant length at which there was direct bone-implant contact without intervening soft tissue layer. The measurements were performed along the whole length of the implant, excluding the most apical part.

(b) Bone mass percentage: The bone mass was evaluated as the percentage of bone present in 2 defined fields: 0-1 $\mathrm{mm}$ and $1-2 \mathrm{~mm}$ from the implant surface.

All measurements were performed for at least 3 sections per implant, and the results were based on the average of these measurements.

\section{Statistical analysis}

All measurements were statistically evaluated using paired t-test for paired data or analysis of variance (ANOVA) and Tukey test for multiple comparisons of the means of different groups. The method of Kolmogorov and Smirnov was used to confirm that the data were sampled from a Gaussian distribution. In case of ANOVA analysis, the method of Bartlett tested the assumption that the standard deviations of the groups were equal. Differences were considered statistically significant when $p$ was $<0.05$.

\section{RESULTS}

\section{Experimental animals}

None of the goats showed signs of weakness after retrieval of blood before surgery. All goats had an uneventful postoperative recovery, and their weight remained stable during the experimental period. At sacrifice, no clinical signs of inflammation or adverse tissue reactions could be seen. Radiographs taken parallel to the long axis of the implant assured that the implants were located in trabecular bone. Although 3 implants appeared to be placed near the epiphyseal growth plate, this did not hamper their histomorphometrical evaluation.

\section{PRP preparation}

The volumes of the PRP and platelet concentrations, as harvested from each goat, are shown in Table 1. According to this randomized study design, goat 1 and goat 4 received implants without application of PRP. The PRP preparation resulted in platelet concentrations ranging from 800 to $1200 \times 10^{6}$ platelets $/ \mathrm{mL}$.

\section{Histological evaluation}

The light microscopical evaluation of the implants showed an uneventful healing of all implants without any sign of inflammatory response. In all sections, the marginal bone was at the same level as the top of the implant. Occasionally, coronal invasion of connective tissue along the implant surface was observed in some sections of all 6 implant groups but never exceeded $2 \mathrm{~mm}$. Because the histomorphometrical measurements were performed along the whole length of the implant, the crestal resorption and connective tissue invasion affected the bone contact percentage negatively. In most sections, the drilling procedure appeared to be accurate. In a limited number of sections, the drill hole was too long. Therefore, the most apical part (bottom) of the implants was excluded from the study. The damage caused by the drilling procedure appeared to be limited, as characterized by the absence of drilling debris or fractured bone particles.

\section{CaP-coated implants without PRP (Fig. 2A)}

The histological sections for all CaP-coated implants revealed a uniform bone reaction. In the interfacial area, new bone formation had occurred along the implant surface, whereas marrow spaces of different volume had interrupted this bone-implant contact. The bone was in close contact with the cement surface, without any sign of intervening fibrous tissue layer. The implants were never associated with an inflammatory response.

\section{CaP-coated implants with PRP liquid (Fig. 2B)}

In the area directly adjacent to the implant, new bone formation had occurred on the implant surface without any evidence of a soft tissue layer. The surface of the implants was almost completely covered with a thin layer of new lamellar bone. At the implant-bone interface, remodeling lacunas containing osteoblasts and bone marrow-like tissue were clearly visible. Next to the implant, no accumulations of inflammatory cells were seen.

\section{CaP-coated implants with PRP gel (Fig. 2C)}

No differences in healing response between this group and the other 2 groups of CaP-coated implants were observed. A tight contact existed between the newly deposited bone and the CaP-coated surface. In the interfacial area, new bone formation had occurred along the implant surface without an intervening fibrous tissue layer. Again, large marrow spaces that contained osteoblasts interrupted this bone-implant contact. No macrophages or giant cells were seen between the implant surface and surrounding bone.

\section{Non-coated implants without PRP (Fig. 3A)}

In this group as well, none of the non-coated titanium implants showed an adverse bone reaction. In the interfacial area, new bone formation occurred along the implant 


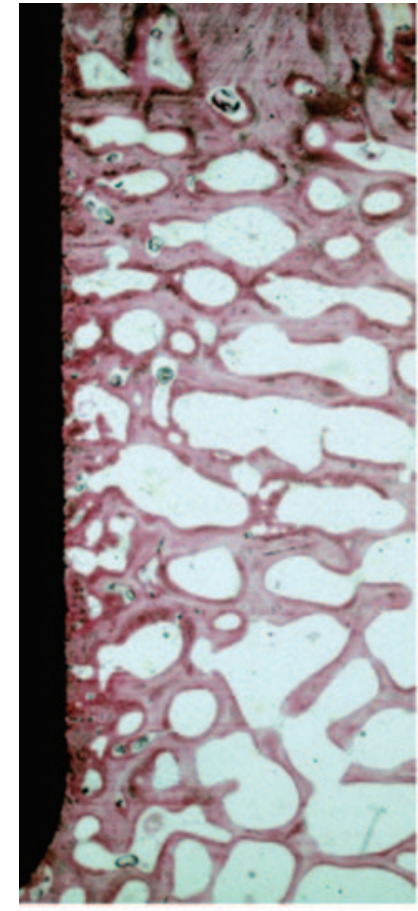

A

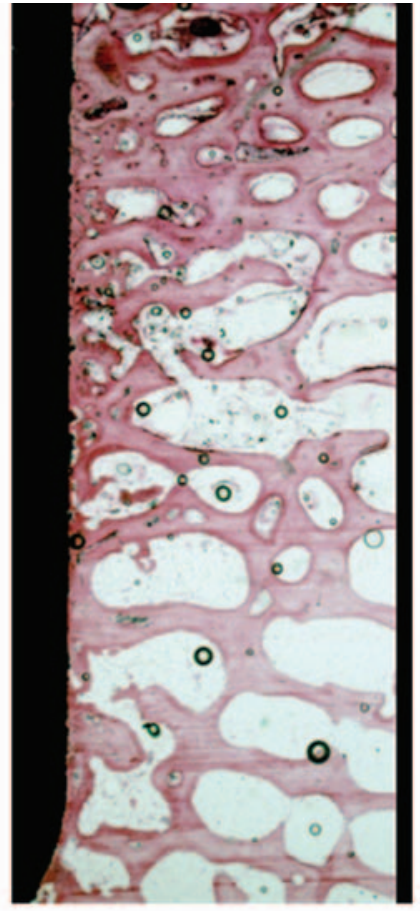

B

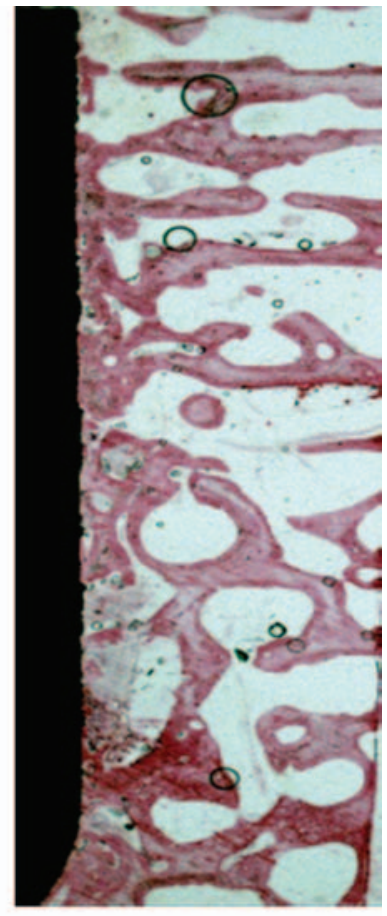

C

FIG. 2. Histological sections of the calcium phosphate-coated titanium implants (magnification 16x): (A) without application of platelet-rich plasma (PRP), (B) with the use of PRP liquid, (C) with the use of PRP gel. Color images available online at www.liebertpub.com/ten.

surface, whereas marrow spaces of different volumes had interrupted this bone-implant contact. The newly formed bone had mainly a lamellar structure. Similar to the other implant groups, no inflammatory cells were seen in any section.

\section{Non-coated implants with PRP liquid (Fig. 3B)}

Bone-apposition and bone-remodeling lacunae with bone marrow stromal cells in close contact with the implant surface were observed. The bone was closely adapted to the implant surface without any sign of fibrous tissue formation. The interfacial bone contact appeared to be more pronounced than the other non-coated implants. The PRP liquid had not evoked an inflammatory response.

\section{Non-coated implants with PRP gel (Fig. 3C)}

In almost all sections, a thin layer of new lamellar bone covered the implant surface without any fibrous tissue intervention. Although in some sections, residual amorphous material, probably consisting of degraded PRP gel, occupied the inter-trabecular bone voids, no inflammatory reaction was observed.

\section{Histomorphometrical evaluation}

Percentage of bone contact: The results of the bone contact measurements are listed in Table 2. The data show that the highest average amount of bone contact (94\%) occurred for the $\mathrm{CaP} /$ liquid implants, whereas the titaniumimplant group showed the lowest amount (60\%). Statistical comparison of the bone contact percentages between the individual implant groups and various treatment combinations confirmed that

1. no significant differences existed in bone contact percentage between the $3 \mathrm{CaP}$ coated-implant groups

2. titanium / liquid implants showed a significantly higher bone contact percentage than the other 2 titanium implant groups

3. no significant differences existed in bone contact percentage between the titanium/liquid and any $\mathrm{CaP}$ coated implant group

Percentage of bone mass: The results for the bone mass measurements are also given in Table 2. The bone mass was determined in 2 different zones: $0-1 \mathrm{~mm}$ and $1-2 \mathrm{~mm}$ from the implant surface. The results show that the percentage of bone mass in the 0 - to $1-\mathrm{mm}$ zone ranged from $49 \%$ to $69 \%$, whereas in the $1-$ to $2-\mathrm{mm}$ zone, it ranged from $34 \%$ to $55 \%$.

Statistical analysis showed that in the $0-$ to $1-\mathrm{mm}$ area all the implant groups induced a similar bone mass except the titanium and titanium/gel implants, which were surrounded 


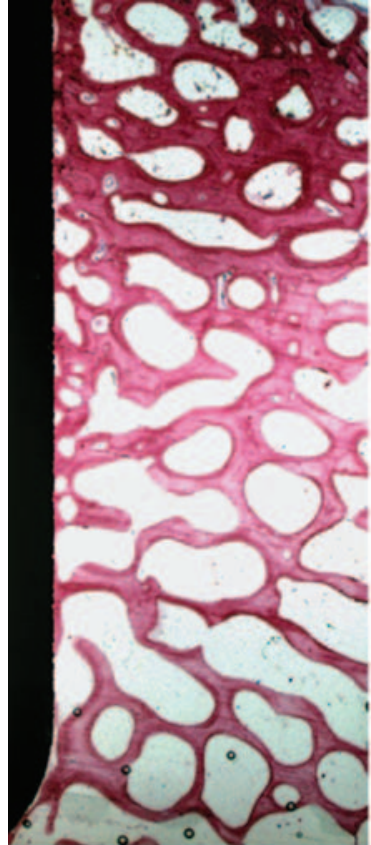

A

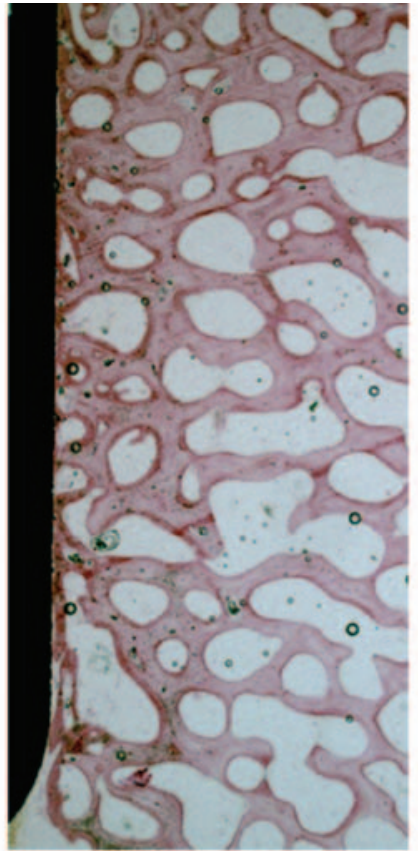

B

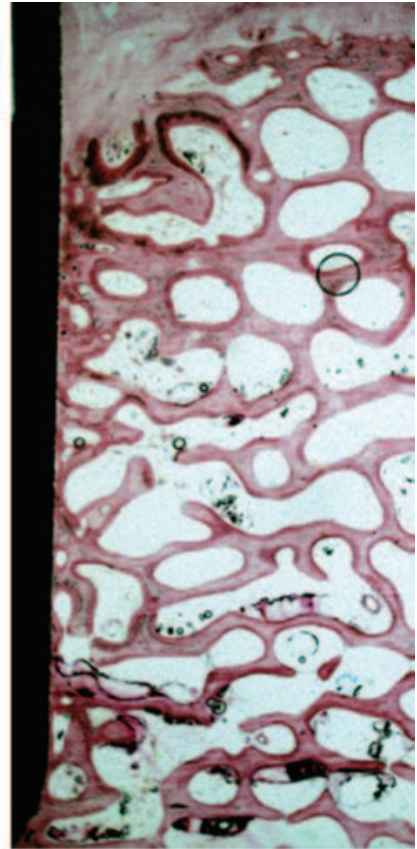

C

FIG. 3. Histological sections of the non-coated rough titanium implants (magnification 16x): (A) without application of platelet-rich plasma (PRP), (B) with the use of PRP liquid, (C) with the use of PRP gel. Color images available online at www.liebertpub.com/ten.

by a significantly lower amount of bone. Also, in the area 1 to $2 \mathrm{~mm}$ away from the implant surface, a lower mass of bone surrounded the titanium group than the other groups. Finally, statistical evaluation (paired t-test) of the bone mass between the 2 zones in each group indicated that, for the $\mathrm{CaP}-, \mathrm{CaP} /$ liquid-, titanium-, and titanium/ liquid-implant groups, more bone mass $(p<0.05)$ was present in the 0 - to $1-\mathrm{mm}$ zone than in the 1 - to $2-\mathrm{mm}$ zone. Only for the implants inserted in combination with the PRP gel did the amount of bone mass not differ significantly $(p>0.05)$ between the 2 zones.

\section{DISCUSSION}

The purpose of the present study was to evaluate the effect of the surface quality ( $\mathrm{CaP}$ coating and surface

Table 2. Mean and Standard Deviation of the Percentage of Bone Contact (\%) and Bone Mass (\%) Estimated in Two Different Zones 0-1 mm and 1-2 mm from the Implant Surface

\begin{tabular}{llccc}
\hline Group & \multicolumn{1}{c}{ Treatment modality } & Bone mass (\%) & $\begin{array}{c}\text { Bone mass (\%) } \\
\text { zone 1-2 mm }\end{array}$ \\
\hline Titanium & Non-coated implant (no PRP) & $60 \pm 9 *$ & $55 \pm 7^{\S}$ & $34 \pm 1^{\#}$ \\
Titanium / liquid & Non-coated implant + PRP liquid & $79 \pm 11$ & $69 \pm 4$ & $51 \pm 7$ \\
Titanium / gel & Non-coated implant + PRP gel & $62 \pm 11$ & $49 \pm 8^{\S}$ & $47 \pm 4$ \\
CaP & CaP-coated implant (no PRP) & $83 \pm 4$ & $62 \pm 4$ & $43 \pm 7$ \\
CaP/liquid & CaP-coated implant + PRP liquid & $94 \pm 4$ & $66 \pm 7$ & $46 \pm 1$ \\
CaP/gel & CaP-coated implant + PRP gel & $81 \pm 10$ & $63 \pm 9$ & $55 \pm 8$ \\
\hline
\end{tabular}

Six separate study groups were created based on the different treatment modalities. Analysis of variance and Tukey-Kramer multiple comparisons test were used for the statistical analysis.

*The statistical analysis revealed that the non-coated/platelet-rich plasma (PRP) and non-coated/PRP gel groups had significantly less bone contact than the other groups $(p<0.05)$. No other difference was significant.

${ }^{\S}$ The statistical analysis revealed that the bone density in the region of $0-1 \mathrm{~mm}$ from the implant was significantly lower in the non-coated/PRP and non-coated / PRP gel groups than in all the other groups $(p<0.05)$.

"The bone density in the region of 1-2 mm from the implant was significantly lower in the non-coated/PRP group than in the non-coated/PRP liquid $(p<0.05)$ and calcium phosphate $(\mathrm{CaP}) /$ PRP gel groups $(p<0.01)$. 
roughness) in combination with the application of PRP on the initial bone response after implant placement.

Non-coated and CaP-coated implants had an almost similar roughness, yet scanning electron microscopy revealed small differences in surface morphology. The additional $\mathrm{CaP}$ coating caused these small differences. The final effect of this disparity on the study outcome can currently not be answered and requires further investigation. Perhaps for future studies, a solution for the surface roughness discrepancy can be found by deposition of an additional thin titanium coating on the roughened bulk titanium implant.

Notwithstanding this limitation in study design, the histological and histomorphometric evaluation demonstrated that the application of a thin magnetron-sputtered $\mathrm{CaP}$ coating (1- to $2-\mu \mathrm{m}$ thickness) favored the early bone response. Six weeks after implantation, a higher percentage of bone-implant contact was established for CaP-coated implants than for the non-coated ones. These findings corroborate previous studies with similar coated implants in the same animal model. ${ }^{9,23}$

Furthermore, the results revealed that PRP liquid (without any activation) favored the bone-implant contact for the roughened titanium implants, because the PRP liquid implant groups always showed a significantly higher percentage of bone contact than the other 2 titanium-implant groups (PRP gel and control). Although there was also a tendency toward greater bone contact for the CaP-coated implants after the application of PRP liquid, this difference was not statistically significant. On the other hand, no effect of the use of PRP gel on bone contact percentage was observed for CaP-coated or non-coated titanium implants. A similar study by Zechner et al. in 2003 in a mini-pig model also failed to show any statistically significant effect of PRP gel on peri-implant bone formation after a 6-week healing period. ${ }^{24}$ In another study, Weibrich et al. analyzed the influence of PRP gel on bone regeneration around selftapping titanium screw implants inserted in the femoral bone of rabbits. ${ }^{25}$ After a 4 -week healing period, no advantageous effect on bone-implant contact rate was seen. These results are in agreement with our findings about PRP gel. An explanation for the difference in result between PRP liquid and PRP gel might be the trauma caused during the installation of the PRP gel. After putting the PRP gel in place, the implant is forced into the implant cavity. PRP gel has a viscous, jelly-like consistency, which can result in additional pressure on the drill walls of the already tightly fitting implant. As a consequence, the healing process at the bone-implant interface might become disturbed. Support for this hypothesis is found in the occurrence of degraded remnants of PRP gel in the inter-trabecular bone voids around the titanium/gel implants. This might also clarify why Kim et al. also found a higher percentage of bone contact 6 weeks after implant placement when using PRP gel, because they used a peri-implant defect model and not a press-fit technique for implant insertion. ${ }^{26}$ In addition, it is an interesting histologic finding that remnants of PRP gel were only found around titanium/gel implants and not around the CaP implants. A possible explanation is that the $\mathrm{CaP}$ coating may play a role in the faster degradation of PRP gel. In a recent study, ${ }^{27}$ the authors suggest that bio-ceramics may enhance macrophage activation.

For the bone mass measurements, the bone mass was calculated into 2 separate zones of 0 to $1 \mathrm{~mm}$ and 1 to $2 \mathrm{~mm}$ from the implant surface. Changes in the former zone can be related to the surgical method and the materials that were used, whereas changes in the latter zone may be related to the bone quality (density) of the implant preparation site. Comparison of the obtained data showed that the 0 - to $1-\mathrm{mm}$ zone around the titanium and titanium/gel implants contained significantly less bone mass than the other implants. Furthermore, the results revealed greater bone density in the 0 - to $1-\mathrm{mm}$ zone than in the 1- to $2-\mathrm{mm}$ zone for all groups except for the implants inserted in combination with the PRP gel. In this case, bone density did not differ significantly $(p>0.05)$ between the 2 zones. An explanation for the difference in results obtained with PRP gel might be the trauma caused during installation of the gel; bone responds to mechanical injury by increasing in volume and density. Slotte et al. estimated a 35\% increase in bone mass after implant placement in comparison with control bony sites in a rabbit model with an 8-week healing period. ${ }^{28}$ Similar results were obtained in our study, because there was evidently a greater increase in bone mass in the 0 - to $1-\mathrm{mm}$ zone than in the $1-$ to $2-\mathrm{mm}$ zone. Nevertheless, the measurements at a distance of 1 to $2 \mathrm{~mm}$ revealed also that the amount of bone mass was not uniform around all implant types. This variation in bone mass between the groups may be an indication of a difference in bone density between the implant preparation sites, between experimental animals, or both. The femoral condyle consists of 2 different sections-the epiphysis and the metaphysis - which have a different bone density and contain different cell populations, leading to different woundhealing events after implantation. Still, the final effect of PRP liquid and implant surface composition was consistent.

\section{CONCLUSIONS}

The results of the present study confirmed that magnetron-sputtered $\mathrm{CaP}$ coatings can improve the integration of oral implants in trabecular bone. The additional use of PRP did not offer any significant effect on the bone response to the CaP-coated implants, whereas PRP in a liquid form had a significant effect on bone apposition to roughened titanium implants during the early post-implantation healing phase. Nevertheless, additional studies are necessary to 
elucidate the underlying phenomenon for this effect and to determine whether this indeed can be attributed to the PRP.

\section{ACKNOWLEDGMENTS}

We would like to thank Jacky den Bakker for help with the histology and Cees Verhagen, Sanquin Zuid-Oost, Nijmegen, The Netherlands, for the PRP preparation.

\section{REFERENCES}

1. Adell, R., Eriksson, B., Lekholm, U., Branemark, P.I., and Jemt, T. Long-term follow-up study of osseointegrated implants in the treatment of totally edentulous jaws. Int J Oral Maxillofac Implants 5, 347, 1990.

2. van Steenberghe, D., Lekholm, U., Bolender, C., Folmer, T., Henry, P., Herrmann, I., Higuchi, K., Laney, W., Linden, U., and Astrand, P. Applicability of osseointegrated oral implants in the rehabilitation of partial edentulism: A prospective multicenter study on 558 fixtures. Int J Oral Maxillofac Implants 5, 272, 1990.

3. Albrektsson, T., and Lekholm, U. Osseointegration: current state of the art. Dent Clin North Am 33, 537, 1989.

4. Fugazzotto, P., Wheeler, S.L., and Lindsay, J.A. Success and failure rates in cylinder implants in type IV bone. J Periodontol 64, 1085, 1993.

5. Lekholm, C.J., and Zarb, G.A. Patient selection and preparation. In: Branemark, P.I., Zarb, G.A., and Albrektsson, T., eds. Tissue Integrated Prostheses. Osseointegration in Clinical Dentistry, 3rd Ed. Chicago: Quintessence Publishing Co., 1985, pp. 199-209.

6. Jaffin, R.A., and Berman, C.L. The excessive loss of Branemark fixtures in type IV bone: a 5-year analysis. J Periodontol 62, 2, 1991.

7. Albrektsson, T., and Wennerberg, A. Oral implant surfaces: Part 1. Review focusing on topographic and chemical properties of different surfaces and in vivo response to them. Int $\mathbf{J}$ Prosthodont 17, 536, 2004.

8. Søballe, K., Hansen, E.S., Brockstedt-Rasmussen, H., Pedersen, C.M., and Bünger, C. Hydroxyapatite coating enhances fixation of porous coated implants. A comparison in dogs between press fit and non-interference fit. Acta Orthop Scand 61, 299, 1990.

9. Vercaigne, S., Wolke, J.G.C., Naert, I., and Jansen, J.A. A histological evaluation of $\mathrm{TiO}_{2}$-gritblasted and $\mathrm{Ca}-\mathrm{P}$ magnetron sputter coated implants placed into the trabecular bone of the goat: part 2. Clin Oral Implants Res 11, 314, 2000.

10. Hayakawa, T., Yoshinari, M., Nemoto, K., Wolke, J.G.C., and Jansen, J.A. Effect of surface roughness and calcium phosphate coating on the implant/bone response. Clin Oral Implants Res 11, 296, 2000.

11. Vehof, J.W.M., Haus, M.T.U., de Ruijter, J.E., Spauwen, P.H.M., and Jansen, J.A. Bone formation in transforming growth factor beta-I-loaded titanium fiber mesh implants. Clin Oral Implants Res 13, 94, 2002.

12. Vehof, J.W.M., Mahmood, J., Takita, H., van't Hof, M.A., Kuboki, Y., Spauwen, P.H.M., and Jansen, J.A. Ectopic bone formation in titanium mesh loaded with bone morphogenetic protein and coated with calcium phosphate. Plast Reconstr Surg 108, 434, 2001.

13. Calabresi, P.A., Fields, N.S., Maloni, H.W., Hanham, A., Carlino, J., Moore, J., Levin, M.C., Dhib-Jalbut, S., Tranquill, L.R., Austin, H., McFarland, H.F., and Racke, M.K. Phase I trial of transforming growth factor beta 2 in chronic progressive MS. Neurology 51, 289, 1998.

14. Marx, R.E., Carlson, E.R., Eichstaedt, R.M., Schimmele, S.R., Strauss, J.E., and Georgeff, K.R. Platelet-rich plasma: growth factor enhancement for bone grafts. Oral Surg Oral Med Oral Pathol Oral Radiol Endod 85, 638, 1998.

15. Ganio, C., Tenewitz, F., Wilson, R.C., and Maules, B.G. The treatment of chronic non-healing wounds using autologous platelet derived growth factors. J Foot Ankle Surg 32, 263, 1993.

16. Anitua, E. Plasma rich in growth factors: preliminary results of use in the preparation of future sites for implants. Int J Oral Maxillofac Implants 14, 529, 1999.

17. Marx, R.E. Platelet-rich plasma (PRP): what is PRP and what is not PRP? Implant Dent 10, 225, 2001.

18. Tözüm, T.F., and Demirapl, B. Platelet-rich plasma: A promising innovation in dentistry. J Can Dent Assoc 69, 664, 2003.

19. Sanchez, A., Sheridan, P., and Kupp, L. Is platelet-rich plasma the perfect enhancement factor? A current review. Int J Oral Maxillofac Implants 18, 93, 2003.

20. Landesberg, R., Moses, M., and Karpatkin, M. Risks of using platelet rich plasma gel. J Oral Maxillofac Surg 56, 1116, 1998.

21. Dugrillon, A., Eichler, H., Kern, S., and Kliiter, H. Autologous concentrated platelet-rich plasma (cPRP) for local application in bone regeneration. Int J Oral Maxillofac Surg 31, 615, 2002.

22. Clemmons, R.M., Bliss, E.L., Dorsey-Lee, M.R., Seachord, C.L., and Meyers, K.M. Platelet function, size and yield in whole blood and in platelet-rich plasma prepared using differing centrifugation force and time in domestic and foodproducing animals. Thromb Haemost 50, 838, 1983.

23. Hulshoff, J.E.G., Hayakawa, T., van Dijk, K., LeijdekkersGovers, A.F.M., van der Waerden, J.P.C.M., and Jansen, J.A. Mechanical and histologic evaluation of Ca-P plasma-spray and magnetron sputter-coated implants in trabecular bone of the goat. J Biomed Mater Res 36, 75, 1997.

24. Zechner, W., Tangl, S., Tepper, G., Furst, G., Bernhart, T., Haas, R., Mailath, G., and Watzek, G. Influence of plateletrich plasma on osseous healing of dental implants: a histologic and histomorphometric study in mini-pigs. Int $\mathrm{J}$ Oral Maxillofac Implants 18, 15, 2003.

25. Weibrich, G., Hansen, T., Kleis, W., Buch, R., and Hitzler, W.E. Effect of platelet concentration in platelet-rich plasma on peri-implant bone regeneration. Bone 34, 665, 2004.

26. Kim, S.G., Kim, W.K., Park, J.C., and Kim, H.J. A comparative study of osseointegration of Avana implants in a 
demineralized freeze-dried bone alone or with platelet-rich plasma. J Oral Maxillofac Surg 60, 1018, 2002.

27. Silva, S.N., Pereira, M.M., Goes, A.M., and Leite, M.F. Effect of biphasic calcium phosphate on human macrophage functions in vitro. J Biomed Mater Res A 65, 475, 2003.

28. Slotte, C., Lundgren, D., Sennerby, L., and Lundgren, A.K. Influence of preimplant surgical intervention and implant placement on bone wound healing. Clin Oral Implants Res 14, $528,2003$.
Address reprint requests to: John A. Jansen, D.D.S., Ph.D. Department of Periodontology and Biomaterials Radboud University Nijmegen Medical Center PO Box 9101 6500 HB Nijmegen, The Netherlands

E-mail: J.Jansen@dent.umcn.nl 
